\title{
Amiloidosi e Glomerulonefrite Extracapillare: un raro caso clinico di overlap
}

\author{
Francesca Travasoni, Mariangela Annoloro, Alessia Bortot, Stefano Cantelli, Giorgia Russo, \\ Yuri Battaglia
}

\author{
U.O.C. di Nefrologia, Azienda Ospedaliero-Universitaria Arcispedale "Sant'Anna”, Ferrara
}

\begin{abstract}
EXTRACAPILLARY GLOMERULONEPHRITIS OVERLAID ON PRIMARY AMYLOIDOSIS AL: CASE REPORT
Abstract. Amyloidosis is a disease characterized by the deposition of extracellular protein called amyloid. There are two types of amiloidosis: primary AL and secondary AA. Renal involvement is frequent and it occasionally presents as renal failure progressive sustained by extracapillary glomerulonephritis. The rare cases reported in the literature are related to amyloidosis secondary to rheumatoid arthritis.

We report case of a 46 year old woman, smoker with autoimmune hypothyroidism , came to our observation for nephrotic syndrome, hypertension, anemia, acute renal failure. Renal biopsy showed extracapillary glomerulonephritis overlaid on primary amyloidosis AL.

Despite the early start of drug therapy, the patient died after about six months.
\end{abstract}

Key words: Amiloidosis AL, Extracapillary glomerulonephritis, Rapidly progressive renal failure

Conflict of interest: None.

Financial support: None.

Ricevuto: 21 Luglio 2013; Accettato: 23 Ottobre 2013

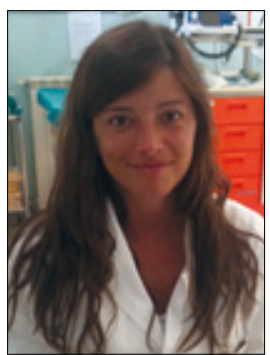

Introduzione

L'amiloidosi è una malattia caratterizzata dalla deposizione in sede extracellulare di materiale proteico a ridotto peso molecolare e insolubile, detto amiloide, che causa disfunzioni d'organo sino alla morte (1). Le fibrille amiloidi hanno una struttura ordinata caratterizzata da precisi criteri diagnostici utilizzati per porre diagnosi di amiloide:

Francesca Travasoni birifrangenza verde mela alla luce polarizzata, colorazione rosso Congo e la tipica struttura fibrillare quaternaria alla microscopia elettronica (2).

Nell'amiloidosi sistemica sia primitiva che secondaria, il coinvolgimento renale è un'evenienza frequente e costituisce la maggiore causa di morbilità di questi pazienti $(3,4)$. L'evoluzione dell'insufficienza renale cronica dagli stadi precoci alla fase predialitica è mediamente di cinque anni. In casi eccezionali, si assiste a un'insufficienza renale rapidamente progressiva sostenuta da una glomerulonefrite extracapillare. I rari casi riportati in letteratura sino a ora sono tutti correlati ad amiloidosi secondarie tipo AA (5). Si riporta il caso clinico di una paziente affetta da amiloidosi renale primitiva AL con insufficienza renale rapidamente progressiva da sovrapposizione di glomerulonefrite extracapillare.

\section{Caso clinico}

Giovane donna di 46 anni con abitudine tabagica (20 sigarette/ die) e di assunzione di alcolici (1/2 litro di vino/die), pregressa sarcoidosi polmonare trattata con terapia steroidea per circa un anno nel 1992, esiti di intervento endoscopico di polipectomia colica e ipotiroidismo secondario a tiroidite di Hashimoto nel 2007 in terapia ormonale sostitutiva.

Nel Gennaio del 2011 effettuava l'accesso in Day Hospital internistico per astenia ingravescente associata a menometrorragie ricorrenti. Al momento del ricovero si documentavano valori di pressione arteriosa pari a 140/80 $\mathrm{mmHg}$, pallore muco-cutaneo, toni cardiaci ritmici, addome trattabile e obiettività neurologica rilevata nella norma. Alla radiografia del torace si documentavano accentuazione del disegno vascolo-interstiziale alle perilari inferiori e iperdiafania del parenchima in assenza di aree definite di addensamento. All'ecografia addominale si riscontravano epatomegalia di grado elevato per steatosi di III grado, pancreas a ecostruttura iperecogena, reni regolari a corticale ben conservata. Agli esami ematochimici si riscontrava anemia microcitica $(\mathrm{Hb}=8.4 \mathrm{~g} / \mathrm{dL}, \mathrm{MCV} 78 \mathrm{fl}$, ferritina $<5 \mathrm{ng} / \mathrm{mL}$, sideremia $21 \mathrm{mcg} / \mathrm{dL}$ ) associata a ipoprotidemia totale (Prot tot $=5.8 \mathrm{mg} / \mathrm{dL})$. Gli indici di funzionalità renale erano nella norma (creatinina $=0.5 \mathrm{mg} / \mathrm{dL}$, eGFR $>90$ $\mathrm{mL} / \mathrm{min}$ ). La ricerca del sangue occulto nelle feci era positiva. 


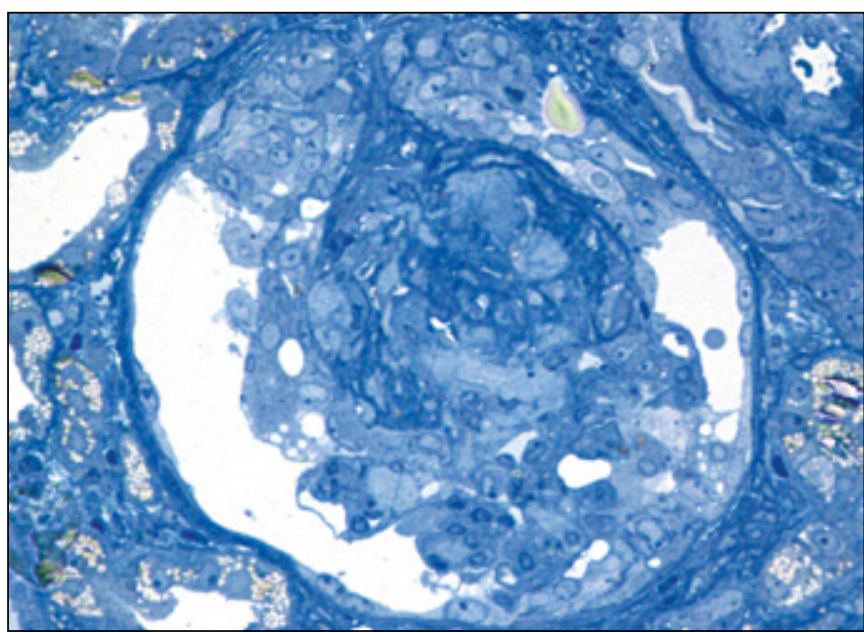

Fig. 1 - Microscopia ottica.

La paziente rifiutava ulteriori approfondimenti diagnostici e iniziava una terapia marziale per via orale.

Nell'Aprile del 2012 la paziente veniva nuovamente ricoverata in regime di DH internistico per la persistenza dell'astenia. Gli esami di laboratorio confermavano il quadro di anemia $(\mathrm{Hb}=$ $10.2 \mathrm{~g} / \mathrm{dL}$ ) microcitica ipocromica associata a carenza marziale e a positività per la ricerca del sangue occulto nelle feci; si confermavano nella norma i parametri di funzionalità renale.

L'approfondimento diagnostico endoscopico colico documentava un quadro di dolicocolon e diverticolosi del sigma in assenza di fonti di sanguinamento in atto. L'esame proctologico evidenziava emorroidi interne di II-III grado congeste, modicamente dolenti. La paziente rifiutava di sottoporsi a ulteriori indagini e proseguiva la terapia marziale al domicilio.

Nel Settembre del 2012 la paziente si recava in Pronto Soccorso a causa della comparsa di edemi declivi colonnari improntabili, associati a contrazione della diuresi e al riscontro di una severa ipertensione arteriosa con valori pari a 180/120 $\mathrm{mmHg}$. Inoltre, la paziente riferiva astenia e iporessia secondarie a nausea e a vomito ricorrenti. Gli esami ematochimici documentavano un aggravamento dello stato anemico $(\mathrm{Hb}=$ $7.5 \mathrm{~g} / \mathrm{dL}$ ) associato a grave insufficienza renale (creatinina = $8.3 \mathrm{mg} / \mathrm{dL}, \mathrm{GFR}=5 \mathrm{~mL} / \mathrm{min}$ ) con marcata ipoalbuminemia (protidemia totale $4.4 \mathrm{~g} / \mathrm{dL}$, albumina $45 \%$ ) e proteinuria in range nefrosico $(3.5 \mathrm{~g} / 24 \mathrm{~h})$. L'elettroforesi proteica evidenziava catene libere sieriche lambda all'immunofissazione pari al $2.4 \%$, migranti in posizione gamma. Il dosaggio della proteinuria di Bence Jones evidenziava valori di catene leggere kappa urinarie pari a $2.7 \mathrm{mg} / \mathrm{dL}$ e di catene leggere lambda urinarie pari a $22.9 \mathrm{mg} / \mathrm{dL}$. L'esame delle urine standard documentava eritrocituria $50 / \mathrm{mcl}$ e leucocituria $416 / \mathrm{mcl}$. Gli esami immunologici (ENA, ANCA, C3, C4, IgG, IgM, IgA, Anti-DNA, Anti-fosfolipidi) sono risultati negativi, tranne ANA (1:320). L'ecografia renale mostrava reni di dimensioni e di spessore corticale conservati, ma maggiormente ipoecogeni rispetto all'indagine ecografica precedente. In considerazione del rapido e progressivo peggioramento della funzione renale, la paziente veniva sottoposta ad agobiopsia renale: la

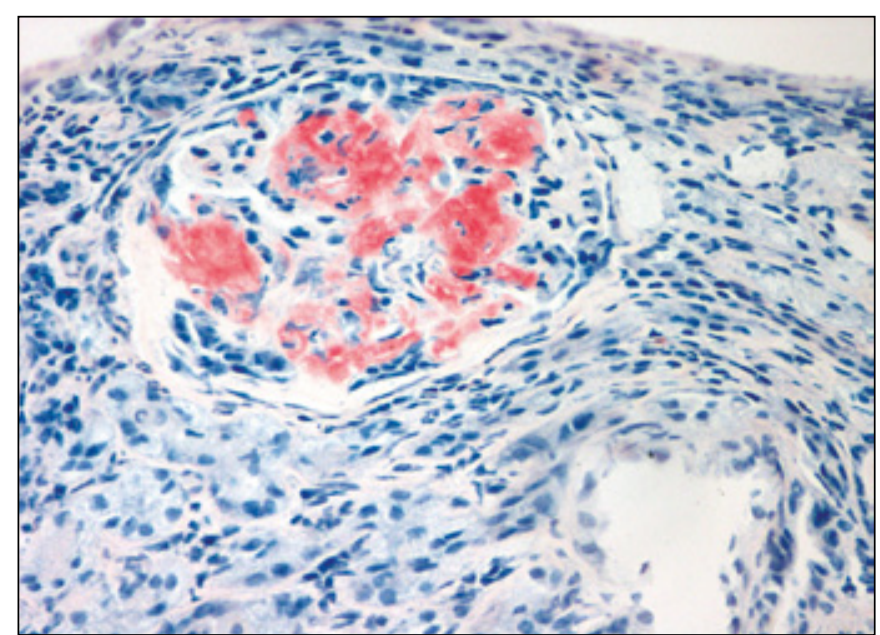

Fig. 2 - Colorazione rosso Congo.

microscopia ottica mostrava sia la presenza di evidenti depositi di materiale amorfo, debolmente eosinofilo, pas positivo, congofilo e dicroico alla luce polarizzata, con ridotta cellularità nel flocculo, sia una proliferazione epiteliale extracapillare, che realizzava immagini a semiluna, compatibili con un quadro di amiloidosi renale e di glomerulonefrite extracapillare (Figg. 1-3).

La tipizzazione dell'amiloide risultava positiva ad anticorpi policlonali anti-catene leggere lambda e negativa risultava l'immunoreazione con anticorpi anti-catene leggere kappa e anti-SAA come da amiloidosi AL. Per il dato anamnestico di sarcoidosi polmonare, la paziente veniva sottoposta anche a tomografia computerizzata toracica con il riscontro di un netto ispessimento pseudonodulare in sede sub pleurica bilateralmente e, successivamente, a broncoscopia con relativo prelievo bioptico risultato positivo per amiloidosi (Fig. 4). Inoltre, la rivalutazione dell'esame istologico con colorazione rosso Congo della mucosa gastrica, prelevato in corso di gastroscopia effettuata all'ingresso per inquadramento diagnostico dello stato anemico, confermava la presenza di amiloide a livello gastrico. Per la stadiazione della malattia veniva effettuata una biopsia midollare che documentava una diseritropoiesi di lieve entità, in assenza di una rilevata e sicura espansione plasma-cellulare, mentre l'ecocardiogramma non mostrava segni di amiloidosi e la frazione di eiezione cardiaca sinistra risultava nei limiti. Dato il quadro di deficit motorio e sensitivo agli arti inferiori, la paziente veniva sottoposta a elettromiografia, che documentava una polineuropatia somatica sensitivo-motoria assonale agli arti inferiori e segni di sofferenza muscolare primitiva, diffusi e disomogenei, prevalenti ai muscoli prossimali e compatibili con la deposizione del materiale amorfo sulle fibre nervose.

A fronte del dato istologico renale si infondeva solumedrol $500 \mathrm{mg} /$ die e.v. in bolo per tre giorni consecutivi con successiva terapia steroidea per via orale a scalare da $0.5 \mathrm{mg} / \mathrm{kg}$ per sei mesi, ottenendo una parziale ripresa della diuresi già dopo il primo bolo. In contemporanea, si somministrava ciclofosfamide al dosaggio di $100 \mathrm{mg} /$ die, sospeso dopo una settimana di trattamento per severa neutropenia, trattata con fattori di 


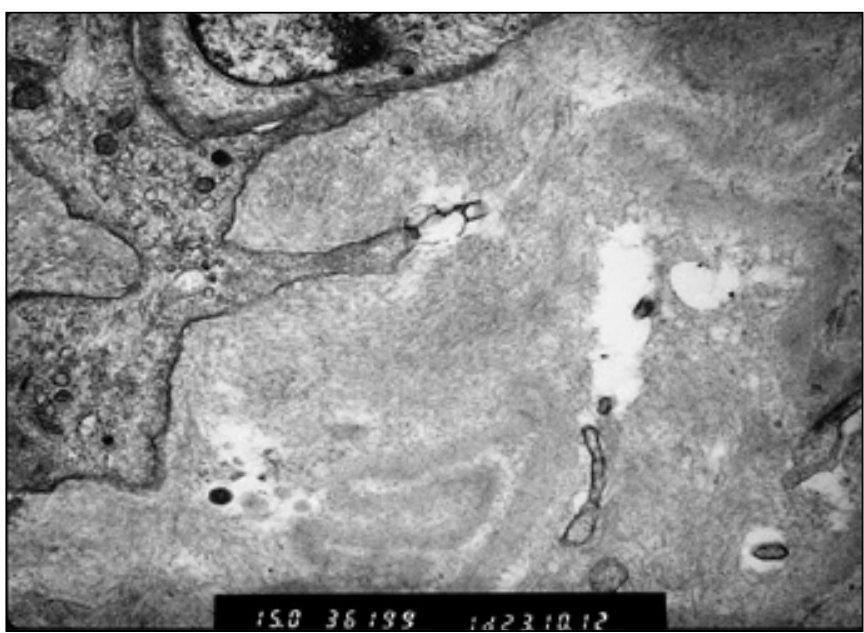

Fig. 3 - Microscopia elettronica.

crescita specifici G-CSF, e infezione delle vie urinarie.

Nel Dicembre del 2012, in accordo con i Colleghi Ematologi, in considerazione del quadro di amiloidosi sistemica primitiva, si iniziava il ciclo di desametasone $8 \mathrm{mg}$ e bortezomib 1.3 $\mathrm{mg} / \mathrm{m}^{2}$ per due volte/settimana per due settimane consecutive in un mese. Al termine del secondo ciclo si era manifestata una cheratite erpetica prontamente risolta con aciclovir e.v. e antiretrovirali per via topica. Sono stati completati sei cicli di terapia.

Nonostante l'avvio tempestivo della terapia immunosoppressiva, gli indici di funzionalità renale erano peggiorati ulteriormente con la contrazione della diuresi, per cui, in data 28 Settembre del 2012, veniva avviato un trattamento emodialitico extracorporeo con ritmo trisettimanale.

Grande difficoltà è stata incontrata nella creazione e nel mantenimento degli accessi vascolari per emodialisi. La paziente è stata sottoposta a ripetuti confezionamenti di FAV native e protesiche e a posizionamenti di CVC in vene giugulari e succlavie, tutti trombizzati a causa della difficoltosa gestione della terapia anticoagulante, a fronte del ripetersi di episodi di sanguinamento a partenza dal tratto gastroenterico (rettorragie, ematemesi), dal tratto respiratorio, nonché dai tratti vaginale e orale. Nel Febbraio del 2013, per impossibilità di reperimento di accessi vascolari, la paziente veniva convertita alla dialisi peritoneale automatizzata notturna.

All'ecocardiogramma di controllo del Marzo del 2013 si riscontrava un aumento degli spessori di parete compatibile con l'iniziale infiltrazione di amiloidosi miocardica (SIV 11.6 $\mathrm{mm}$, PP $11.6 \mathrm{~mm}$, FE 71\%).

Nei mesi successivi si riscontrava un peggioramento delle condizioni cliniche generali: marcata astenia con importante calo ponderale, parestesie a calza, in particolare alla pianta dei piedi, porpora alle palpebre, dispnea per sforzi lievi, ipostenia agli arti inferiori e deambulazione solo per pochi passi. Nell'Aprile del 2013 la paziente è deceduta.

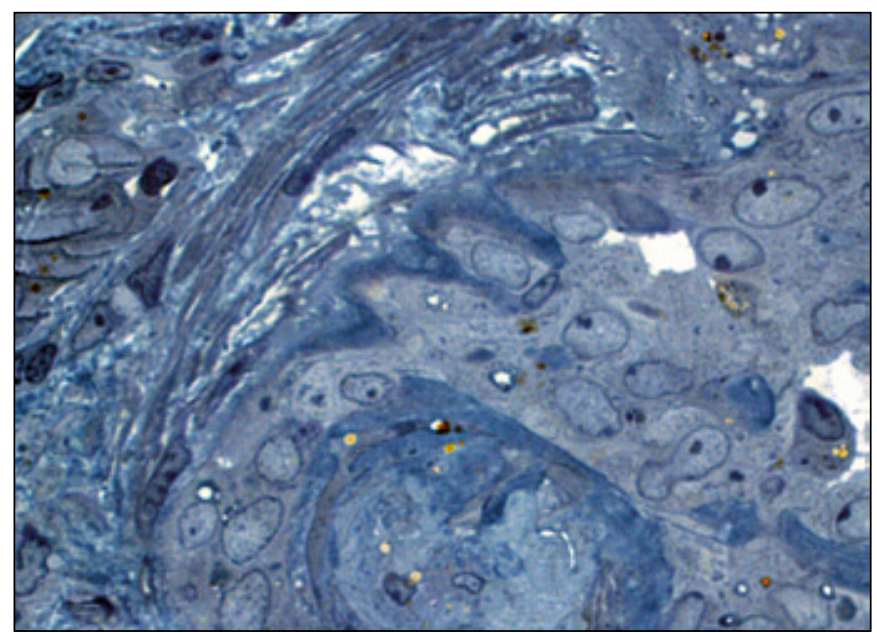

Fig. 4 - Amiloidosi polmonare.

\section{Discussione}

I primi quattro casi di sovrapposizione di glomerulonefrite extracapillare nell'ambito dell'amiloidosi furono descritti da Watanabe et al. che, all'esame istologico, identificarono la deposizione di fibrille amiloidi nello spazio sub endoteliale. Ipotizzarono che i depositi di amiloide avrebbero potuto alterare l'integrità morfologica della membrana basale capillare, creando punti di debolezza per lesioni meccaniche o ischemiche con la conseguente formazione di semilune (5).

Nel 1996, Moroni et al. descrissero tre casi clinici di amiloidosi AA con coinvolgimento renale supportato da un reperto bioptico di sovrapposta glomerulonefrite extracapillare. Due dei pazienti descritti ebbero una buona risposta clinica alla terapia di combinazione con steroidi ad alte dosi e agenti immunosoppressori. Il terzo paziente sospese precocemente la terapia con ciclofosfamide per l'insorgenza di un'artrite settica, andando incontro a insufficienza renale terminale in 2 mesi (6). In letteratura sono riportati altri cinque casi di amiloidosi renale con insufficienza renale rapidamente progressiva sostenuta da glomerulonefrite extracapillare. I cinque pazienti, trattati precocemente con una terapia immunosoppressiva, ottennero una buona risposta clinica. Quattro pazienti erano affetti da amiloidosi secondaria ad artrite reumatoide, mentre il quinto era verosimilmente affetto da amiloidosi $\mathrm{AL}$, anche se in assenza di tipizzazione immunoistochimica dell'amiloide $(7,8)$.

Dalla letteratura, quindi, non emergono casi clinici certi di sovrapposizione di glomerulonefrite extracapillare nell'ambito dell'amiloidosi primitiva.

Inoltre, è evidente che un trattamento aggressivo con corticosteroidi e farmaci immunosoppressivi può dare un beneficio clinico nei pazienti con glomerulonefrite extracapillare associata ad amiloidosi renale (6). Nel nostro caso clinico, nonostante il trattamento tempestivo effettuato con metilprednisolone $500 \mathrm{mg} /$ die in boli per tre giorni e ciclofosfamide 100 $\mathrm{mg} /$ die per via orale, non si è ottenuta alcuna risposta clinica. Non è stato possibile valutare l'andamento delle catene leggere libere circolanti, data la mancanza di un dosaggio prima 
dell'inizio della terapia. Le cause di questo fallimento terapeutico sono, a nostro avviso, molteplici: una patogenesi differente (amiloidosi AL), il mancato completamento del ciclo con ciclofosfamide per la comparsa di neutropenia e infezioni dopo una settimana di terapia e un quadro evolutivo di amiloidosi molto aggressivo.

Altro punto importante di riflessione è l'opportunità di effettuare come prima indagine diagnostica l'agobiopsia renale nel sospetto di un'amiloidosi sistemica. Alcuni studi dimostrano che la biopsia renale può essere gravata da un elevato rischio di complicanze emorragiche, data la carenza del fattore $X$ associato all'amiloidosi $(9,10)$ e consigliano l'utilizzo di mezzi meno invasivi, come l'aspirazione del grasso periombelicale $(11,12)$. Tuttavia, nei casi in cui si manifesta un rapido deterioramento della funzione renale in corso di amiloidosi, è opportuno eseguire la biopsia renale nell'intento di cercare una glomerulonefrite extracapillare, potenzialmente reversibile con un trattamento adeguato.

\section{Conclusioni}

La presenza di una glomerulonefrite extracapillare in un quadro di amiloidosi renale costituisce un'evenienza molto rara ma possibile, non solo in forme di amiloidosi secondarie ad artrite reumatoide ma anche in forme primitive AL. L'esecuzione della biopsia renale in pazienti con amiloidosi che sviluppano un quadro di insufficienza renale rapidamente progressiva risulta mandatoria per rilevare la presenza di un'eventuale glomerulonefrite extracapillare e per avviare terapie farmacologiche adeguate che risultano spesso efficaci.

\section{Riassunto}

L'amiloidosi è una malattia caratterizzata dalla deposizione in sede extracellulare di materiale di natura proteica detto amiloide. Questa malattia sistemica può essere sia primiti- va AL che secondaria AA. Il coinvolgimento renale è un'evenienza frequente e, occasionalmente, si assiste a un'insufficienza renale rapidamente progressiva sostenuta dalla sovrapposizione di una glomerulonefrite extracapillare. I rari casi riportati in letteratura sono correlati a un'amiloidosi secondaria all'artrite reumatoide.

Si riporta il caso clinico di una donna di 46 anni tabagista, affetta da ipotiroidismo su base autoimmune, giunta alla nostra osservazione per la comparsa di sindrome nefrosica, ipertensione arteriosa, anemizzazione e insufficienza renale acuta. L'esame bioptico renale evidenziava un quadro compatibile con la presenza contemporanea di amiloidosi primitiva AL e glomerulonefrite extracapillare. Nonostante l'avvio tempestivo della terapia farmacologica, la paziente è deceduta dopo circa sei mesi.

Parole chiave: Amiloidosi AL, Glomerulonefrite extracapillare, Insufficienza renale rapidamente progressiva

Dichiarazione di conflitto di interessi: Gli Autori dichiarano di non avere conflitto di interessi.

Contributi economici agli Autori: Gli Autori dichiarano di non aver ricevuto sponsorizzazioni economiche per la preparazione dell'articolo.

Indirizzo degli Autori:

Dr. Yuri Battaglia

U.O.C. di Nefrologia

Azienda Ospedaliero-Universitaria Arcispedale "Sant'Anna" Via Aldo Moro 8

44124 Cona (FE)

battagliayuri@gmail.com

\section{Bibliografia}

1. Kyle RA. Amyloidosis: a convoluted story. Br J Haematol 2001; 114: 529-38.

2. Glenner GG. Amyloid deposits and amyloidosis. The beta-fibrilloses (first of two parts). N Engl J Med 1980; 302: 1283-92.

3. Dember LM. Amyloidosis-associated kidney disease. J Am Soc Nephrol 2006; 17: 3458-71.

4. Kyle RA, Greipp PR. Amyloidosis (AL). Clinical and laboratory features in 229 cases. Mayo Clin Proc 1983; 58: 665-83.

5. Watanabe T, Toyosima H, Nagafuchi Y, Yoshikawa Y. Renal amyloidosis with crescents. Hum Pathol 1984; 15: 684-6.

6. Moroni G, Banfi G, Maccario M, Mereghetti M, Ponticelli C. Extracapillary glomerulonephritis and renal amyloidosis. Am J Kidney Dis 1996; 28 (5): 695-9.

7. Kiyama S, Sakemi T, Shimokama T, Baba N, Watanabe T. Crescentic glomerulonephritis associated with renal amyloidosis.
Jpn J Med 1991; 30: 238-42.

8. Diaz Gallo C, Mauri JM, Poveda R, et al. Renal amyloidosis associated with antiglomerular basement membrane disease. Nephron 1990; 56: 335-6.

9. Furie B, Greene E, Furie BC. Factor X deficiency and systemic amyloidosis: In vivo studies of the metabolic fate of factor X. N Engl J Med 1977; 297: 81-5.

10. Yood RA, Skinner M, Rubinow A, Talarico L, Cohen AS. Bleeding manifestations in 100 patients with amyloidosis. JAMA 1983; 249: 1322-4.

11. Gertz MA, Li CY, Shirahama T, Kyle RA. Subcutaneous fat aspiration for the diagnosis of systemic amyloidosis (immunoglobulin light chain). Arch Intern Med 1988; 148: 929-33.

12. Dhingra S, Krishnani N, Kumari N, Pandey R. Evaluation of abdominal fat pad aspiration cytology and grading for detection in systemic amyloidosis. Acta Cytol 2007; 51: 860-4. 


\section{TEST di VERIFICA}

\section{1) La glomerulonefrite extracapillare si può sovrapporre a:}
a) Amiloidosi $\mathrm{AL}$
b) Amiloidosi AA
c) Amiloidosi AA e AL
d) Nessuna delle precedenti

\section{2) La biopsia renale in caso di sospetto di amiloidosi:}

a) È sempre necessaria

b) Non si effettua mai

c) Può essere effettuata come prima indagine diagnostica

d) Deve essere effettuata soltanto dopo la biopsia del grasso periombelicale
3) Il trattamento della glomerulonefrite extracapillare in corso di amilodosi:

a) Non si effettua dati i gravi effetti collaterali che può determinare sull'amiloidosi

b) Prevede schemi terapeutici specifici

c) La terapia immunosoppressiva non ha alcun effetto

d) Si può effettuare con cortisone e ciclofosfamide 